\title{
Zn-Coordinated Lipid Nanocapsules with High Physical Stability and Water-Responsive Morphological Change
}

\author{
Wuxiao Ding, Masaru Aoyagi, Mitsutoshi Masuda and Masaki Kogiso* \\ Research Institute for Sustainable Chemistry, National Institute of Advanced Industrial Science and Technology (AIST), Tsukuba Central 5, 1-1-1 \\ Higashi, Tsukuba, Ibaraki 305-8565 JAPAN
}

\begin{abstract}
A novel lipid nanocapsule with high physical stability and the ability to undergo a waterresponsive morphological change was prepared using a facile method from inexpensive and simple materials such as a glycylglycine-containing lipid and zinc acetate. The zinc-coordinated nanocapsule is comprised of solid bilayer membranes, which are stabilized by polyglycine-II type hydrogen-bond network and $\mathrm{Zn}$-lipid coordination resulting in a high thermal stability in the dry state. The nanocapsules can easily encapsulate guest molecules such as methylene blue in the hollow space by dissolving the molecules in ethanol during preparation. When placed in an aqueous environment, the nanocapsules showed distinctive morphological changes and subsequent release of the methylene blue.
\end{abstract}

Key words: nanocapsule, lipid, nanovesicle, self-assembly, stimuli-response

\section{Introduction}

Nanocapsules like lipid nanovesicles have shown practical applications in medicines, food, pesticides, and dailycare products $^{1-3)}$. They can deliver not only hydrophobic agents embedded in their hydrophobic membrane, but also hydrophilic agents encapsulated in the closed nanospace ${ }^{4,5)}$. Amphiphilic phospholipids and surfactants self-assembled into nanovesicles with a liquid crystalline bilayer membrane, where the hydrocarbon chains assemble through a weak hydrophobic interaction ${ }^{6-8)}$. This weak interaction was detrimental to their morphological stability and drug retention ability above room temperature $\left(25^{\circ} \mathrm{C}\right)$ that resulted from membrane fluidity ${ }^{9,10)}$. Therefore, the physical stability of the nanovesicles is an important problem to be investigated. To date, stabilization techniques such as the addition of preserving agents or crosslinking of the bilayer membrane have been developed ${ }^{9,11,12)}$.

In the meanwhile, the lipid nanovesicles can be designed to be unstable under specific conditions, called stimuli-response $^{13)}$. The nanovesicles respond to stimuli through different mechanisms such as membrane destabilization, opening of pores on the membrane, and by morphological transformations ${ }^{14-17)}$. Since the physical stability and stim- uli-response of the self-assembled nanovesicles were both directed by non-covalent intermolecular interactions ${ }^{18,19)}$, a precise design of these non-covalent intermolecular interactions is necessary for achieving a balanced physical stability and stimuli-response. To the best of our knowledge, lipid nanovesicles possessing both features have not yet been reported. Such nanovesicles are expected to have advantages such as retention of their morphology for longterm storage and ability for on-demand release of encapsulated molecules.

We previously reported that simple glycylglycine-containing lipids formed metal-coordinated nanotubes in an ethanol-water mixture $^{20,21)}$. Hydrogen bonding and metal coordination increased the physical stability of the lipid bilayers, and also triggered a morphological change from plates into nanotubes. These nanotubes have been shown to be potential drug carriers at both the cellular level and in animals ${ }^{22,23)}$. However, encapsulation of the drug was inefficient since the cylindrical space within the nanotubes is open.

Here, we report on the selective and quantitative formation of a metal-coordinated lipid nanocapsule with a vesicular structure in ethanol(Fig. 1). The structure was evaluat-

\footnotetext{
* Correspondence to: Masaki Kogiso, Research Institute for Sustainable Chemistry, National Institute of Advanced Industrial Science and Technology (AIST), Tsukuba Central 5, 1-1-1 Higashi, Tsukuba, Ibaraki 305-8565 JAPAN

E-mail: m-kogiso@aist.go.jp

Accepted August 30, 2016 (received for review July 8, 2016)

Journal of Oleo Science ISSN 1345-8957 print / ISSN 1347-3352 online

http://www.jstage.jst.go.jp/browse/jos/ http://mc.manusriptcentral.com/jjocs
} 


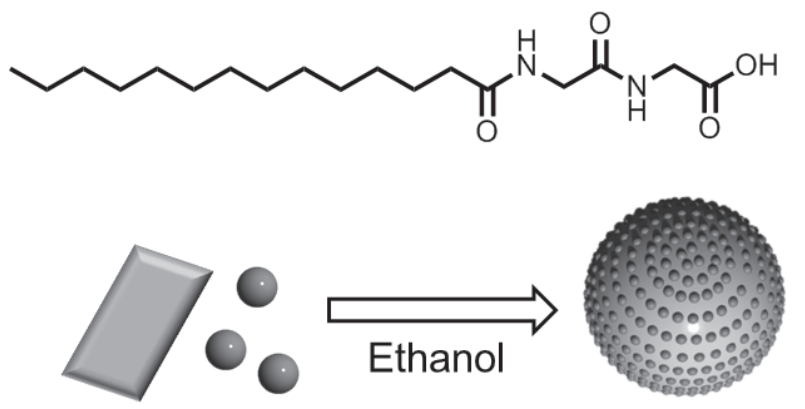

Plate $\mathrm{Zn}^{2+}$

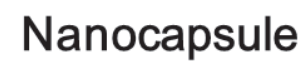

Fig. 1 Schematic illustration of the nanocapsule by mixing the plate made of glycylglycine-containing lipid with zinc ions in ethanol.

ed by Fourier transform infrared spectroscopy(FT-IR), $\mathrm{X}$-ray diffraction (XRD), and thermogravimetric analyses (TGA). The physical stabilities at $25^{\circ} \mathrm{C}$ and at an elevated temperature were investigated. Finally, the encapsulation of functional molecules such as methylene blue and its release accompanying a morphological change were also investigated.

\section{Materials and Methods}

\subsection{Materials}

The lipid was synthesized as previously reported[20]. Zinc acetate dihydrate, ethanol (99.5 vol\%), and methylene blue (MB) were purchased from Wako Pure Chemical Industries, Ltd., and used without further purification.

\subsection{Preparation and characterization of the $\mathrm{Zn}$-coordinat- ed nanocapsules}

Zinc acetate dihydrate $(0.549 \mathrm{~g}, 2.5 \mathrm{mmol})$ solution in ethanol (30 mL) was added into the lipid ( $1.71 \mathrm{~g}, 5 \mathrm{mmol})$ dispersion in ethanol $(10 \mathrm{~mL})$ at room temperature. After 4 $\mathrm{h}$, the resultant Zn-coordinated nanocapsules were collected by filtration and dried in vacuum. The morphology of the nanocapsules was confirmed by scanning transmission electron microscopy (STEM, Hitachi S-4800). The detailed structure was characterized by FT-IR, XRD, and TGA analyses.

\subsection{Encapsulation and release of methylene blue (MB)}

A model guest, MB, was added into the lipid dispersion before the addition of zinc acetate dihydrate. After reacting for $4 \mathrm{~h}$, free MB was completely removed by filtration and washed with ethanol until the filtrate turned colorless. The MB-encapsulated nanocapsules were dried in vacuum and granulated into fine powders for measuring the encapsulation efficiency (the weight ratio of MB to the lipid) and the release profile.
One hundred milligrams of MB-encapsulated nanocapsules was vigorously dispersed separately in $10 \mathrm{~mL}$ of water and phosphate buffered saline (PBS buffer) ( $\mathrm{pH}$ 7.4). The dispersions were stirred at $200 \mathrm{rpm}$ at $37^{\circ} \mathrm{C}$. At $10 \mathrm{~min}$, $30 \mathrm{~min}, 1 \mathrm{~h}, 4 \mathrm{~h}, 7 \mathrm{~h}$, and $22 \mathrm{~h}, 1 \mathrm{~mL}$ of the dispersions was sampled and filtered through syringe filters (pore size 450 $\mathrm{nm}$ ) for measuring the absorbance at $663 \mathrm{~nm}$.

\section{Results and Discussion}

3.1 Formation of $\mathrm{Zn}$-coordinated nanocapsule and its physical stability in the dry state.

As-synthesized lipid solid dispersed in ethanol has a plate morphology. The addition of zinc acetate dihydrate into the lipid/ethanol dispersion exclusively transformed it into a sphere after reacting for $4 \mathrm{~h}$ (Fig. 2). The sphere shows a homogenous diameter of $120 \pm 10 \mathrm{~nm}$. Since the spherical lipid aggregates seem to have hollow space (Fig. 2B), they might be a vesicle or a reverse vesicle. The metal-coordinated nanotubes formed in a water-alcohol dispersion have hydrophilic metal-carboxylate groups on the outside, since they showed catalytic activity ${ }^{24)}$. The hollow sphere should be a vesicle, since it had FT-IR spectrum and XRD almost identical to those of the metal-coordinated nanotubes ${ }^{21)}$. Compared to multiple processes needed for the preparation of liposomes ${ }^{1)}$ and metal-coordinated nanovesicles as previously reported ${ }^{25-27)}$, the present nanocapsules show advantages such as the one-step synthesis of lipids and the one-step formation of the homoge-

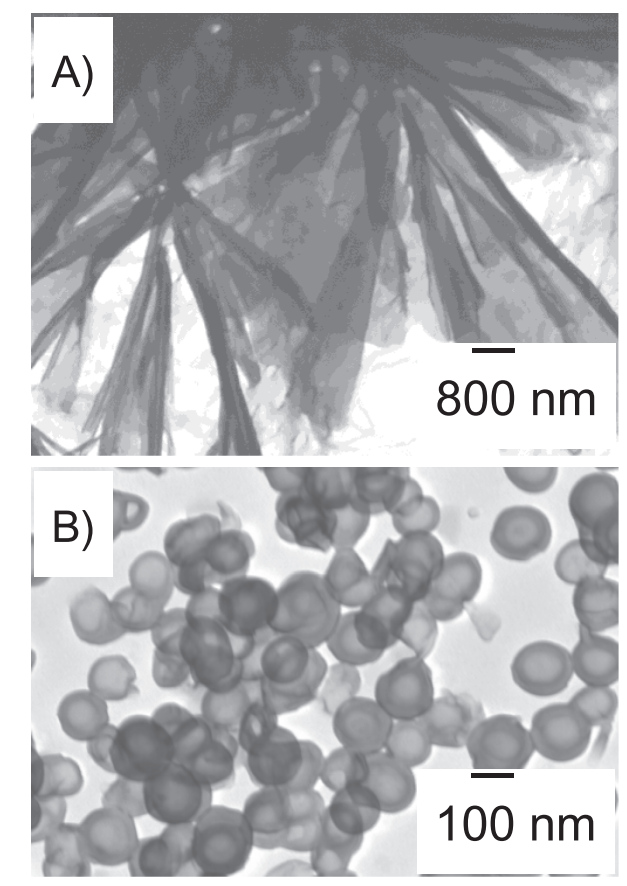

Fig. 2 STEM images of (A) original plate made of lipid and (B) Zn-coordinated nanocapsule. 
neous nanocapsule. Based on the preparation conditions described in the previous section, mass production (such as kilograms at a 10 L-reaction scale) of the nanocapsule is foreseeable.

To investigate the physical stability, the dry nanocapsules were mounted on the carbon grid and stored in air for 1 month at $25^{\circ} \mathrm{C}$ or for $20 \mathrm{~min}$ at $120^{\circ} \mathrm{C}$. STEM observations revealed that the vesicular morphology was strongly retained under both conditions (Fig. S1). Compared with phospholipid nanovesicles that usually show a phase transition temperature lower than $65^{\circ} \mathrm{C}$ and need preserving agents to retain their morphology in the dry state ${ }^{9,28)}$, the present nanovesicles have advantages of high physical stability up to at least $120^{\circ} \mathrm{C}$ in a dry state without using any preserving agents.

\subsection{Structural characterization of the $\mathrm{Zn}$-coordinated nanocapsules}

Structural characterization of the nanocapsules was carried out using FT-IR, XRD, and TGA (Fig. 3). The Zncoordinated nanocapsules showed almost the same FT-IR spectrum and XRD as those of the nanotubes, which were prepared in the ethanol-water mixture ${ }^{20,21)}$. In the FT-IR of the nanocapsules, two peaks at 1652 and $1665 \mathrm{~cm}^{-1}$ indicated the formation of zinc-carboxylate complexes. Two peaks at 1637 and $1025 \mathrm{~cm}^{-1}$ ascribable to the amide I band and a skeletal vibrational band of the glycine moiety, respectively, indicated that the glycylglycine residues formed polyglycine-II type hydrogen-bond network ${ }^{29}$. XRD of the nanocapsules showed periodic diffractions in the small angle region, indicating a solid bilayer repeat distance of $4.6 \mathrm{~nm}$. Since the membrane thickness of the nanocapsules is $20-25 \mathrm{~nm}$ as estimated from STEM images, the membrane consists of 4-5 bilayers. Due to the relatively high crystalline nature, homogeneous nanocapsules could be produced. Such strong interactions and the thick solid bilayer membrane will contribute to the high physical stability of the nanocapsules.

TGA analysis clarified the difference between the nanocapsules and the nanotubes (Fig. 3). TGA of the nanotubes showed a $4.3 \%$ weight loss over $88^{\circ} \mathrm{C}$, which corresponds to a loss of two water molecules. In contrast, the nanocapsules showed no significant weight loss before $230^{\circ} \mathrm{C}$, suggesting that the nanocapsules did not contain any coordinated water molecules. Elemental analyses of the nanocapsules and the nanotube also supported these results. Both two nanostructures are formed from one zinc and two lipids. While the nanotube contains two water molecules, the nanocapsule did not have any water molecules (Table S1).

\subsection{Release of MB from the nanocapsules with morpho- logical changes}

For the given conditions, the encapsulation efficiency of
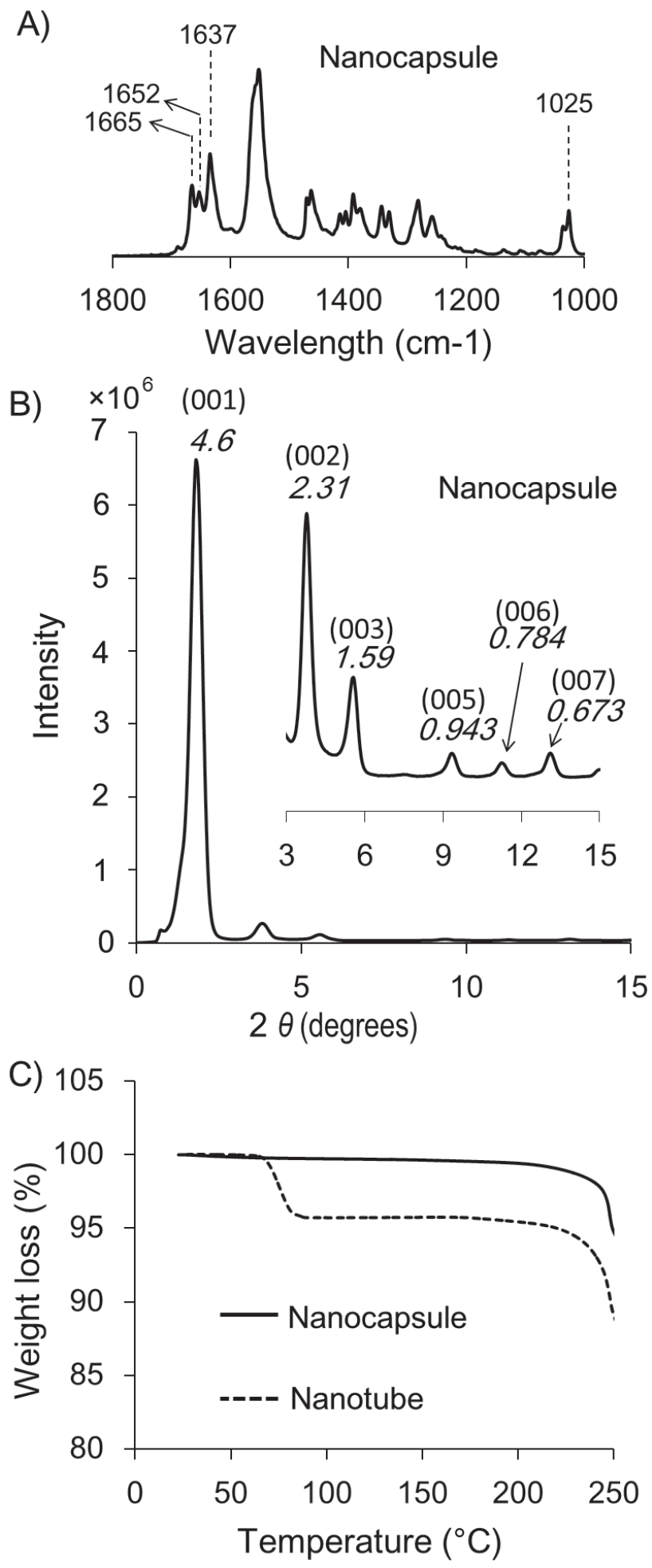

Fig. 3 (A)FT-IR, (B) XRD, and TGA analyses for the characterization of the nanocapsule.

MB molecules in the nanocapsules was found to be around $8.7 \%$, with MB primarily being confined to the closed nanospace. In water, the release of MB from the nanocapsules was slow; only $12 \%$ of MB was released at $22 \mathrm{~h}$ (Fig. 

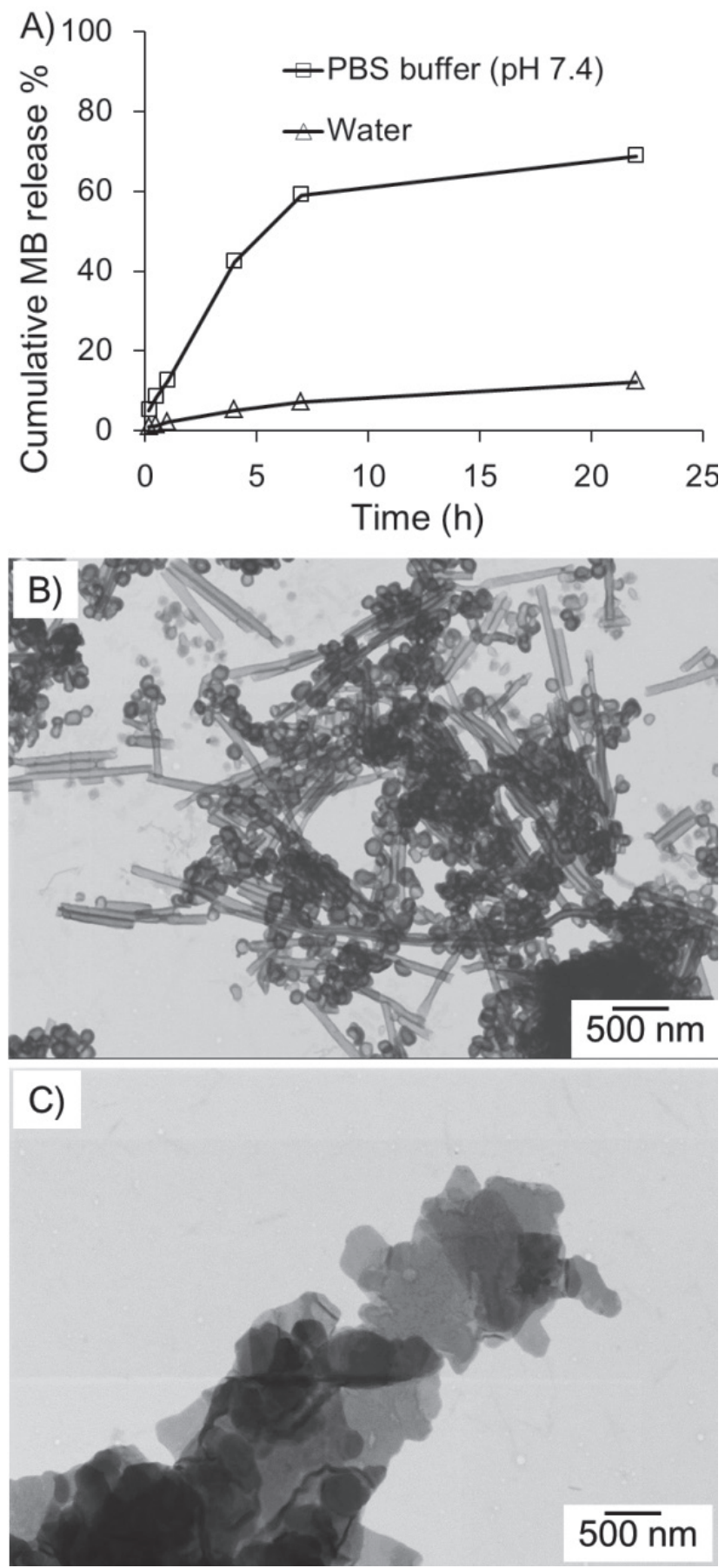

Fig. 4 (A) Release of MB from the nanocapsule, and their morphologies at $22 \mathrm{~h}$ in (B) water and (C) PBS buffer.

4A). A morphological change into the nanotube was partly observed at $22 \mathrm{~h}$ in water (Fig. 4B). We considered that the release of MB mainly occurred during the morphological change from the nanocapsules into the open-ended nanotube. Based on the difference of the nanotube and the nanocapsules (Fig. 3C), we suppose that the hydration of zinc ions may be a driving force for the morphological change of the nanocapsules into the nanotube. In organic solvents like acetone and toluene, the nanocapsules retain their morphology even after one day.

In the PBS buffer, the release of MB from the nanocapsules was obviously facilitated, reaching $68 \%$ at $22 \mathrm{~h}$ (Fig. 4A). A complete morphological transformation into a sheet was confirmed at $22 \mathrm{~h}$ in PBS buffer(Fig. 4C). Since the outer d orbital of zinc (II) is completely filled $\left(d^{10}\right)$, the zinccarboxylate coordination bond is not as strong as that formed with other transition metals. Because of an abundance of ions in the PBS buffer, an equilibrium between zinc and sodium should be attained easily, resulting in a faster morphological change and release than samples dispersed in pure water. Unlike the morphological transformation of the nanocapsules into nanotubes in water, the change in the morphology of the nanocapsules into a sheet in PBS buffer might proceed with a change in the lipid-Zn complex, such as a partial replacement of the zinc ions by sodium ions (Fig. S2).

\section{Conclusions}

A novel lipid nanocapsule with high physical stability and the ability to undergo a water-responsive morphological change was prepared using a facile method. In the absence of any preserving agents, the dry nanocapsules showed high physical stability for potential long-term storage of at least up to 1 month at room temperature. In pure water, the nanocapsules underwent a morphological change into a nanotube, which resulted in a sustained release of the encapsulated methylene blue. The nanocapsules transformed into a sheet and facilitated the release in PBS buffer. The Zn-coordinated nanocapsules may be useful for the sustained as well as stimuli-responsive release of drugs, in pesticides, flavoring agents, and polymers, although the transformation mechanism is still under investigation.

\section{Acknowledgements}

This work was partly supported by JSPS-KAKENHI 24550226.

\section{Supporting Information}

This material is available free of charge via the Internet at http://dx.doi.org/jos.65.10.5650/jos.ess.16132

\section{References and notes}

1) Samad, A.; Sultana, Y.; Aqil, M. Liposomal drug deliv- 
ery systems: an update review. Curr. Drug Deliv. 4, 297-305 (2007).

2) Taylor, T.M.; Davidson, P.M.; Bruce, B.D.; Weiss, J. Liposomal nanocapsules in food science and agriculture. Crit. Rev. Food Sci. Nutr. 45, 587-605(2005).

3) Antonietti, M.; Förster, S. Vesicles and Liposomes: A Self-Assembly Principle Beyond Lipids. Adv. Mater. 15, 1323-1333 (2003).

4) Schwendener, R.A.; Schott, H. Liposome formulations of hydrophobic drugs. Methods Mol. Biol. 605, 129138 (2010).

5) Szoka, F.; Papahadjopoulos, D. Procedure for preparation of liposomes with large internal aqueous space and high capture by reverse-phase evaporation. Proc. Natl. Acad. Sci. USA 75, 4194-4198(1978).

6) Bangham, A.D.; Standish, M.M.; Watkins, J.C. Diffusion of univalent ions across the lamellae of swollen phospholipids. J. Mol. Biol. 13, 238-252 (1965).

7) Papahadjopoulos, D.; Miller, N. Phospholipid model membranes. I. Structural characteristics of hydrated liquid crystals. Biochim. Biophys. Acta 135, 624-638 (1967).

8) Mahale, N.B.; Thakkar, P.D.; Mali, R.G.; Walunj, D.R.; Chaudhari, S.R. Niosomes: novel sustained release nonionic stable vesicular systems--an overview. Adv. Colloid Interface Sci. 183-184, 46-54(2012).

9) Stark, B.; Pabst, G.; Prassl, R. Long-term stability of sterically stabilized liposomes by freezing and freezedrying: Effects of cryoprotectants on structure. Eur. J. Pharm. Sci. 41, 546-555(2010).

10) Armengol, X.; Estelrich, J. Physical stability of different liposome compositions obtained by extrusion method. J. Microencapsul. 12, 525-535(1995).

11) Crowe, L.M.; Crowe, J.H.; Rudolph, A.; Womersley, C.; Appel, L. Preservation of freeze-dried liposomes by trehalose. Arch. Biochem. Biophys. 242, 240-247 (1985).

12) Joo, K.I.; Xiao, L.; Liu, S.; Liu, Y.; Lee, C.L.; Conti, P.S.; Wong, M.K.; Li, Z.; Wang, P. Crosslinked multilamellar liposomes for controlled delivery of anticancer drugs. Biomaterials 34, 3098-3109 (2013).

13) Ganta, S.; Devalapally, H.; Shahiwala, A.; Amiji, M. A review of stimuli-responsive nanocarriers for drug and gene delivery. J. Controled Release 126, 187-204 (2008).

14) Lee, M.; Lee, S.J.; Jiang, L.H. Stimuli-Responsive Supramolecular Nanocapsules from Amphiphilic Calixarene Assembly. J. Am. Chem. Soc. 126, 12724-12725 (2004).

15) Park, C.; Lee, I.H.; Lee, S.; Song, Y.; Rhue, M.; Kim, C. Cyclodextrin-covered organic nanotubes derived from self-assembly of dendrons and their supramolecular transformation. Proc. Natl. Acad. Sci. USA 103, 1199-1203(2006).
16) Yan, X.; He ,Q.; Wang, K.; Duan, L.; Cui, Y.; Li, J. Transition of cationic dipeptide nanotubes into vesicles and oligonucleotide delivery. Angew. Chem. Int. Ed. 46, 2431-2434(2007).

17) Xing, P.; Chen, H.; Bai, L.; Zhao, Y. Photo-triggered transformation from vesicles to branched nanotubes fabricated by a cholesterol-appended cyanostilbene. Chem. Commun. 51, 9309-9312(2015).

18) Whitesides, G.M.; Mathias, J.P.; Seto, C.T. Molecular self-assembly and nanochemistry: a chemical strategy for the synthesis of nanostructures. Science 254, 1312-1319 (1991).

19) Busseron, E.; Ruff, Y.; Moulin, E.; Giuseppone, N. Supramolecular self-assemblies as functional nanomaterials. Nanoscale 5, 7098-7140 (2013).

20) Kogiso, M.; Zhou, Y.; Shimizu, T. Instant Preparation of Self-Assembled Metal-Complexed Lipid Nanotubes That Act as Templates to Produce Metal-Oxide Nanotubes. Adv. Mater. 19, 242-246 (2007).

21) Kogiso, M.; Aoyagi, M.; Asakawa, M.; Shimizu, T. Semisolid Phase Synthesis of Metal-complexed Organic Nanotubes. Chem. Lett. 39, 822-823(2010).

22) Wakasugi, A.; Asakawa, M.; Kogiso, M.; Shimizu, T.; Sato, M.; Maitani, Y. Organic nanotubes for drug loading and cellular delivery. Int. J. Pharm. 413, 271-278 (2011).

23) Maitani, Y.; Nakamura, Y.; Kon, M.; Sanada, E.; Sumiyoshi, K.; Fujine, N.; Asakawa, M.; Kogiso, M.; Shimizu, T. Higher lung accumulation of intravenously injected organic nanotubes. Int. J. Nanomedicine 8, 315-323 (2013).

24) Chattopadhyay, T.; Kogiso, M.; Aoyagi, M.; H. Yui; Asakawa, M.; Shimizu, T.; Single bilayered organic nanotubes: anchors for production of a reusable catalyst with nickel ions. Green Chem. 13, 1138-1140 (2011).

25) Gruber, B.; Kataev, E.; Aschenbrenner, J.; Stadlbauer, S.; König, B. Vesicles and Micelles from Amphiphilic Zinc (II)-Cyclen Complexes as Highly Potent Promoters of Hydrolytic DNA Cleavage. J. Am. Chem. Soc. 133, 20704-20707 (2011)

26) Xing, L.B.; Yu, S.; Wang, X.J.; Wang, G.X.; Chen, B.; Zhang, L.P.; Tung, C.H.; Wu, L.Z. Reversible multistimuli-responsive vesicles formed by an amphiphilic cationic platinum(II) terpyridyl complex with a ferrocene unit in water. Chem. Commun. 48, 10886-10888 (2012).

27) Liu, J.; Morikawa, M.A.; Kimizuka, N. Conversion of Molecular Information by Luminescent Nanointerface Self-Assembled from Amphiphilic Tb (III) Complexes. J. Am. Chem. Soc. 133, 17370-17374(2011).

28) Jing, L.; Wang, X.; Zhang, T.; Wang, C.; Huang, Z.; Luo, X.; Deng, Y. A review on phospholipids and their main applications in drug delivery systems. Asian J. 
W. Ding, M. Aoyagi and M. Masuda et al.

Pharm. Sci. 10, 81-98(2015).

29) Shimizu, T.; Kogiso, M.; Masuda, M. Noncovalent Formation of Polyglycine II-Type Structure by Hexagonal
Self-Assembly of Linear Polymolecular Chains. J. Am. Chem. Soc. 119, 6209-6210(1997). 